\title{
Direct observation of doping incorporation pathways in self-catalytic GaMnAs nanowires
}

\author{
T. Kasama, ${ }^{1, a)}$ M. Thuvander, ${ }^{2}$ A. Siusys,${ }^{3}$ L. C. Gontard,${ }^{4}$ A. Kovács, ${ }^{5}$ S. Yazdi, ${ }^{1}$ \\ M. Duchamp, ${ }^{5}$ A. Gustafsson, ${ }^{6}$ R. E. Dunin-Borkowski, ${ }^{5}$ and J. Sadowski ${ }^{3,7}$ \\ ${ }^{1}$ Center for Electron Nanoscopy, Technical University of Denmark, DK-2800 Kongens Lyngby, Denmark \\ ${ }^{2}$ Department of Applied Physics, Chalmers University of Technology, SE-41296 Gothenburg, Sweden \\ ${ }^{3}$ Institute of Physics, Polish Academy of Sciences, al. Lotników 32/46, PL-02-668 Warszawa, Poland \\ ${ }^{4}$ Instituto de Ciencia de Materiales de Sevilla (CSIC-US), C/Américo Vespucio 49, 41092 Seville, Spain \\ ${ }^{5}$ Ernst Ruska-Centre for Microscopy and Spectroscopy with Electrons and Peter Grünberg Institute, \\ Forschungszentrum Jülich, D-52425 Jülich, Germany \\ ${ }^{6}$ Solid State Physics and the Nanometer Structure Consortium, Lund University, P.O. Box 118, SE-221 00 \\ Lund, Sweden \\ ${ }^{7}$ MAX-IV Laboratory, Lund University, P.O. Box 118, SE-221 00 Lund, Sweden
}

(Received 15 April 2015; accepted 19 July 2015; published online 3 August 2015)

\begin{abstract}
Doping mechanisms of Mn in GaAs nanowires (NWs) that have been grown self-catalytically at $600^{\circ} \mathrm{C}$ by molecular beam epitaxy (MBE) are investigated using advanced electron microscopy techniques and atom probe tomography. $\mathrm{Mn}$ is found to be incorporated primarily in the form of non-magnetic tetragonal $\mathrm{Ga}_{0.82} \mathrm{Mn}_{0.18}$ nanocrystals in Ga catalyst droplets at the ends of the NWs, while trace amounts of $\mathrm{Mn}(22 \pm 4$ at. ppm) are also distributed randomly in the NW bodies without forming clusters or precipitates. The nanocrystals are likely to form after switching off the reaction in the MBE chamber, since they are partially embedded in neck regions of the NWs. The $\mathrm{Ga}_{0.82} \mathrm{Mn}_{0.18}$ nanocrystals and the low $\mathrm{Mn}$ concentration in the $\mathrm{NW}$ bodies are insufficient to induce a ferromagnetic phase transition, suggesting that it is difficult to have high Mn contents in GaAs even in 1-D NW growth via the vapor-liquid-solid process. (C) 2015 AIP Publishing LLC.

[http://dx.doi.org/10.1063/1.4927623]
\end{abstract}

\section{INTRODUCTION}

Diluted magnetic semiconductors (DMSs) are of great interest for the development of future spintronic devices that combine the two major information technologies of semiconductors for logic and magnetism for memory. ${ }^{1}$ Mn-doped GaAs (GaMnAs) was first reported by Ohno et $a l^{2}$ as a promising material system for DMS applications. Recently, the highest reported value for $\mathrm{T}_{\mathrm{c}}$ of $\sim 190 \mathrm{~K}$ has been measured. ${ }^{3}$ In GaMnAs, the Mn atoms that occupy Ga lattice sites act as acceptors, providing not only localized magnetic moments but also holes that mediate ferromagnetic coupling. ${ }^{4}$ In order to obtain a GaMnAs solid solution that exhibits a paramagnetic-to-ferromagnetic phase transition, a Mn concentration exceeding $\sim 1 \%$ is required. ${ }^{4}$

Methods that require high temperatures during crystal growth (bulk crystals or epitaxially growth single crystalline layers ${ }^{5,6}$ ) often cause inherent phase segregation or MnAs nanocrystal formation when the Mn concentration exceeds $\sim 0.1$ at. \%. As a result of the very low solubility of $\mathrm{Mn}$ in GaAs, therefore, it is necessary to use non-equilibrium growth methods such as low-temperature molecular beam epitaxy (MBE), ${ }^{2}$ below $\sim 300^{\circ} \mathrm{C}$ or $\mathrm{Mn}$ ion implantation into GaAs. ${ }^{7}$ However, the samples grown by these methods contain usually a variety of point defects, such as As antisites, ${ }^{8}$ Ga vacancies, ${ }^{9}$ and Mn interstitials, ${ }^{10}$ which degrade their semiconductive and magnetic properties.

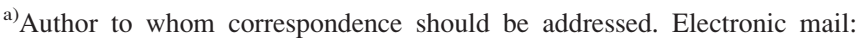
tk@cen.dtu.dk
}

Although the growth of monocrystalline GaMnAs layers using a high Mn flux is impossible at high temperatures, ${ }^{11} 1$ D nanowire (NW) growth at a $\mathrm{Mn} / \mathrm{Ga}$ flux ratio of several percent is possible, which has been demonstrated in several studies. ${ }^{11-14}$ Although some studies have shown room temperature ferromagnetism, ${ }^{14,15}$ the origin and mechanism of the reported magnetization are not clear. For example, the formation of ferromagnetic precipitates or clusters such as MnAs should be considered as a possible source of magnetism. ${ }^{11,16,17}$ Recently, core-shell structured NWs consisting of $\mathrm{GaAs} / \mathrm{GaMnAs},{ }^{18} \mathrm{GaAs} / \mathrm{MnAs},{ }^{19,20}$ and $\mathrm{InGaAs} / \mathrm{GaMnAs}^{21}$ have been considered as well. Some of these studies showed that low temperature grown GaMnAs shells can contain high Mn concentrations up to $\sim 5 \%$ and have ferromagnetic phase transitions above $20 \mathrm{~K}^{18,21}$

We have demonstrated previously ${ }^{11,13}$ that it is possible to grow GaMnAs NWs by MBE at low temperatures $\left(300-350^{\circ} \mathrm{C}\right)$ and if the growth temperature is slightly too high, the formation of MnAs nanoclusters occurs on the surface. However, due to high disorder in terms of NW orientation, crystallographic defects, and morphology, other growth regimes of Mn-doped GaAs NWs have been exploited: (i) Ga catalyzed NWs grown at high temperature on $\mathrm{Si}(100),{ }^{13}$ (ii) Ga catalyzed NWs grown at high temperature on $\mathrm{Si}(111),{ }^{22}$ and (iii) low-temperature grown GaMnAs shells on high-temperature grown Au-catalyzed GaAs NWs on $\operatorname{GaAs}(111) \mathrm{B} .{ }^{21}$ In this paper, we focus on investigating in details structural and compositional properties of Mn-doped Ga catalyzed GaAs NWs grown on $\mathrm{Si}(111)$ at high 
TABLE I. Geometries and growth parameters of the self-catalyzed Mn-doped and undoped GaAs NWs investigated in the present study. Brackets show standard deviations.

\begin{tabular}{lccccc}
\hline \hline Sample & $\begin{array}{c}\text { Nanowire } \\
\text { length (nm) }\end{array}$ & $\begin{array}{c}\text { Nanowire } \\
\text { width (nm) }\end{array}$ & $\begin{array}{c}\text { Ga droplet } \\
\text { diameter (nm) }\end{array}$ & $\begin{array}{c}\text { Growth } \\
\text { rate (nm/s) }\end{array}$ & Growth parameters \\
\hline NW1 (Mn-doped GaAs) & $4950(729)$ & $170(34)$ & $320(61)$ & $0.8(0.1)$ & $\begin{array}{r}\text { GaAs at } 630^{\circ} \mathrm{C} \text { for } 0.25 \mathrm{~h} \text {, followed by GaMnAs } \\
\text { at } 600^{\circ} \mathrm{C} \text { for } 1.5 \mathrm{~h}(5 \mathrm{~s} \mathrm{GaMnAs}+20 \mathrm{~s} \mathrm{GaAs} \times 216 \text { times })\end{array}$ \\
NW2 (Mn-doped GaAs) & $3000(543)$ & $160(32)$ & $290(57)$ & $0.4(0.1)$ & $\begin{array}{r}\text { GaAs at } 630^{\circ} \mathrm{C} \text { for } 0.25 \mathrm{~h}, \text { followed by GaMnAs at } 600^{\circ} \mathrm{C} \\
\text { for } 2 \mathrm{~h}(10 \mathrm{~s} \mathrm{GaMnAs}+20 \mathrm{~s} \mathrm{GaAs} \times 240 \text { times })\end{array}$ \\
NW3 (undoped GaAs) & $3260(563)$ & $200(27)$ & $350(51)$ & $0.4(0.1)$ & GaAs at $630^{\circ} \mathrm{C}$ for $0.25 \mathrm{~h}$, followed by GaAs at $600{ }^{\circ} \mathrm{C}$ for $2 \mathrm{~h}$ \\
\hline \hline
\end{tabular}

${ }^{\mathrm{a}}$ Modulation doping.

temperature $\left(600^{\circ} \mathrm{C}\right)$, classified to (ii). In this case, the NWs would not be expected to reach the Mn content high enough to enable the ferromagnetic phase transition observed in GaMnAs DMSs because of its high growth temperature. However, since Mn has been considered as a possible p-type dopant in GaAs NWs, it is desirable to exploit the Mndoping limits.

Here, we use various quantitative transmission electron microscopy (TEM) techniques and laser-assisted atom probe tomography (LA-APT) in order to study quantitative dopant distributions and dopant incorporation pathways in GaMnAs NWs grown self-catalytically at $600{ }^{\circ} \mathrm{C}$ by MBE. To the best of our knowledge, it is the first report describing detailed Mn incorporation into GaMnAs NWs through their direct observation techniques. Recently, a similar work has been reported on $\mathrm{Mg}$-doped GaN NWs grown with Au catalyst by chemical vapor deposition, showing a significant amount of $\mathrm{Mg}$ was incorporated through the $\mathrm{Au} / \mathrm{GaN}$ interface during the growth. ${ }^{23}$ Gas et $a l .{ }^{22}$ have studied on optical and structural properties of similar GaMnAs NWs to those used in the present study and found a Mn-containing nanocrystal in the single NWs. In this paper, we also investigate the nanocrystals in morphological, crystallographic, compositional, and magnetic structures as well as their formation mechanism, since the phase has not been reported and could be common in GaMnAs NWs. In addition, the dopant concentration measured by LA-APT is compared with that obtained by optical method shown in Ref. 22.

\section{EXPERIMENTAL DETAILS}

Initial growth of GaAs NWs was carried out at $630{ }^{\circ} \mathrm{C}$ on $\mathrm{Si}$ (100) substrates that had 5-nm-thick oxidized surface layers by means of MBE. Mn modulation doping at $600{ }^{\circ} \mathrm{C}$ for 1.5 and $2 \mathrm{~h}$ was then used to grow NW1 and NW2, respectively. The $\mathrm{As}_{2} / \mathrm{Ga}$ flux ratio was kept close to 0.5 during growth in order to obtain NWs with the composition of stoichiometric GaAs. During the doping the Mn/Ga flux ratio was kept close to $3 \%$. When NW growth was terminated, the temperature was decreased to room temperature at cooling rates of $30-40^{\circ} \mathrm{C} / \mathrm{min}$. At the same time, the sources of the target elements were switched off. A small amount of As was expected to remain in the growth chamber after closing the As shutter. For comparison, undoped GaAs NWs (NW3) were prepared under similar conditions. The samples investigated in the present study and their growth parameters are summarized in Table I.
TEM and scanning TEM (STEM) techniques, including electron tomography and off-axis electron holography, were applied to the samples using two FEI Titan 80-300ST fieldemission-gun TEMs operated at either 120 or $300 \mathrm{kV}$. Elemental mapping was performed using energy dispersive X-ray spectroscopy (EDS) in the STEM mode. Each EDS spectrum was acquired typically with a dwell time of $5 \mathrm{~s}$ in steps of $10 \mathrm{~nm}$ and was processed by principal component analysis (PCA) ${ }^{24}$ and pixel-by-pixel background subtraction. Electron diffraction was performed using a high-angle tripleaxis (HATA) TEM specimen holder to obtain as many diffraction patterns as possible from low-index zone axes. The holder allows a specimen to be tilted in a range of $\pm 80^{\circ}$ about the longitudinal axis with tilting capability up to $\pm 7^{\circ}$ about the orthogonal axis. ${ }^{25}$ For electron tomography, tilt series of images were acquired using high-angle annular dark field (HAADF) STEM over a tilt range of $-65^{\circ}$ to $+65^{\circ}$ with a tilt increment of $2^{\circ}$. The inner detector angle used was $57 \mathrm{mrad}$. Off-axis electron holograms were recorded in Lorentz mode (with the objective lens switched off and the specimen in magnetic-field-free conditions) at room temperature and at $100 \mathrm{~K}$. Mean inner potential contributions to the recorded phase shift were removed by using in-situ magnetization reversal. ${ }^{26}$ For comparison, electron holographic magnetic induction maps were calculated using the approach reported by Beleggia and Zhu, ${ }^{27}$ which has been used for the prediction of possible magnetic configurations. ${ }^{28,29}$ For most of the TEM experiments, specimens were prepared by scratching the surface of the NWs-growing substrates with a holey carbon TEM grid. Nanoprecipitates located inside the catalyst particles at the ends of the NWs and GaAs in neck regions of the NWs were characterized in specimens prepared using focused ion beam (FIB) milling. The NWs, which were protected by electronbeam induced $\mathrm{Pt}$ deposition and subsequent ion-beam induced deposition, were fixed onto TEM grids using in-situ lift-out method. The TEM lamellae thinned by FIB milling were polished at $500 \mathrm{~V}$ using low-energy Ar ion milling.

Cathodoluminescence measurements were performed at $8 \mathrm{~K}$ in a scanning electron microscope (SEM) with an accelerating voltage of $5 \mathrm{kV}$ and a beam current of $10 \mathrm{pA}$.

LA-APT experiments were carried out using an Imago LEAP 3000X HR in laser pulse mode with a wavelength of $532 \mathrm{~nm}$. The experimental parameters used are given in the caption to Fig. 7. For LA-APT, single NWs were transferred onto $\mathrm{W}$ needles in-situ in a SEM and fixed onto the needles using electron-beam deposition of Pt or W. 


\section{RESULTS AND DISCUSSION}

Figure 1 shows representative electron micrographs recorded from self-catalyzed GaMnAs and undoped GaAs NWs grown using the conditions given in Table I. The NWs are oriented parallel to the $\langle 111\rangle$ directions of the Si (100) substrate. The NWs each comprise a GaAs body with a cubic zinc-blende structure and a non-crystalline Ga droplet at its end. The formation of the zinc-blende GaAs appears to be more preferable in self-catalyzed GaMnAs NWs with a low surface energy of a $\mathrm{Ga}$ droplet, which can suppress the nucleation of the hexagonal wurtzite GaAs forming at the triple phase line where the Ga liquid droplet is in contact with both vapor and the edge of the NW top surface. ${ }^{30-32}$ All of the NWs contain significant numbers of twins, which usually have higher densities closer to the tops of the wires (Fig. 1). Measurements of NW and droplet diameters are plotted as a function of NW length in Fig. 1 and show that there is a tendency for longer NWs to have wider bodies and larger Ga droplets. The NW diameters increase gradually along their
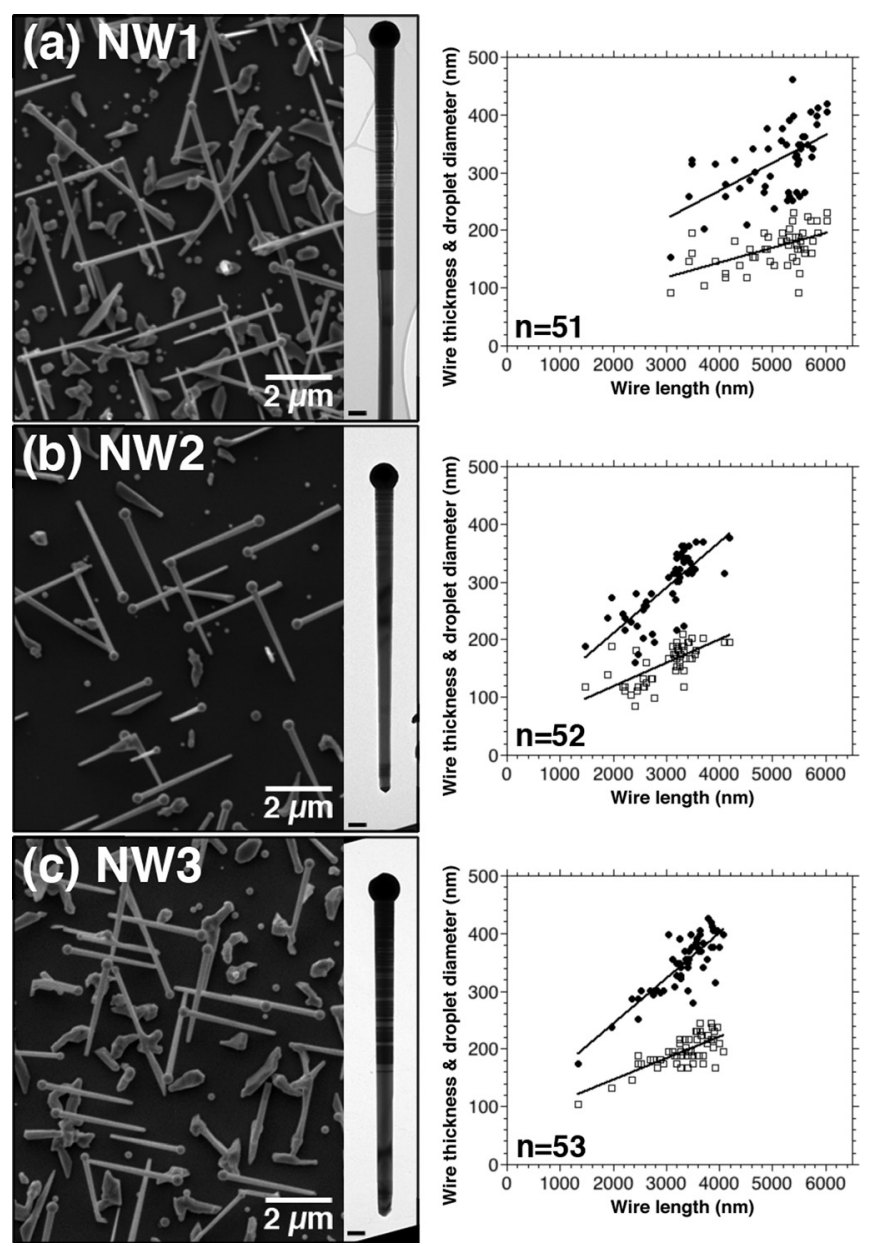

FIG. 1. Representative SEM (left) and TEM (right) images acquired from samples (a) NW1 (GaMnAs), (b) NW2 (GaMnAs), and (c) NW3 (undoped $\mathrm{GaAs}$ ) and the measurements of NW diameter and Ga droplet diameter plotted as a function of NW length for their samples. The measurements were performed using TEM images, and the NW diameters were measured from their middles. Open-square symbols correspond to measurements of NW diameter, while closed-circle symbols represent measurements of Ga droplet diameter. The lines are drawn for eye guide. The scale bars in the TEM images are $100 \mathrm{~nm}$. length as seen in Fig. 1. The growth rates estimated from their mean lengths and growth times are in the range of $0.4-0.8 \mathrm{~nm} / \mathrm{s}$.

Several growth mechanisms have recently been proposed for group III-V compound semiconductor NWs. ${ }^{33}$ Our measurements support particle-assisted growth scheme with seed particles containing elements constituting the NW because the measured NW length and diameter increase with an increase in Ga droplet size (Fig. 1). This scheme is based on the vapor-liquid-solid (VLS) growth mechanism, involving a catalytic liquid droplet adsorbing elements in vapor and becoming supersaturated in the liquid phase, leading to crystallization of the $\mathrm{NW}$ at the droplet/NW interface. Growth directly on the surfaces of the NW bodies without catalytic reaction (i.e., vapor-solid growth) ${ }^{34}$ would be expected to lead to tapered NW growth. Therefore, the VLS growth is considered to be predominant for the present NWs. The increase in droplet size during growth is thought to result from an imbalance between the consumption of $\mathrm{Ga}$ and As by the NWs and their supply from the vapor.

The geometrical characteristics of the NWs in samples NW2 and NW3 are similar, while the NWs in sample NW1 are longer but have similar diameters and droplet sizes to the other wires. The difference between their NW lengths is not presently understood and is not considered in the discussion presented below. It is notable that the use of Mn doping does not affect the sizes, shapes, or crystallographic features of the NWs measurably.

Figure 2 shows quantitative STEM-EDS elemental maps recorded from a GaMnAs NW taken from specimen NW2. $\mathrm{Mn}$ is found to be located with a specific distribution in the Ga droplet, indicating the formation of a nanocrystal, which has been reported previously. ${ }^{22}$ The nanocrystal has significant amounts of Ga and Mn but does not contain As. Seven NWs were examined and all exhibited similar Mn distributions, although the Mn accumulations had smaller volumes in NWs taken from sample NW1, which should be associated with the use of a small amount of Mn for their growth.

In order to fully characterize the individual (Ga,Mn)containing nanocrystals, FIB milling was used to remove much of the surrounding non-crystalline $\mathrm{Ga}$, as shown in Fig. 3. The electron diffraction pattern from a nanocrystal shown in Fig. 3(a) and the high-resolution STEM-HAADF image shown in Fig. 3(c) have both four-fold symmetry, with the diffraction pattern containing all possible $h \mathrm{kl}$ reflections, suggesting that the crystal has a primitive tetragonal lattice. The lattice parameters measured from several diffraction patterns including [001], [011], and [010] zone axes are $a=0.796( \pm 0.015) \mathrm{nm}$ and $c=0.645( \pm 0.013) \mathrm{nm}$. Further observations indicate the absence of $h h l$ reflections with $l=2 n+1$, the absence of $h 0 l$ reflections with $l=2 n+1$ or $h+l=2 n+1$, and the presence of all $h 00$ reflections, suggesting that the (Ga,Mn)-containing phase is likely to be tetragonal with either $P 4 / m c c$ or $P 4 c c$ symmetry. The composition of the nanocrystal is determined by quantitative EDS analysis to be $\mathrm{Ga}_{0.82 \pm 0.03} \mathrm{Mn}_{0.18 \pm 0.03}$ (Fig. 3(d)). The nanocrystal has a strong crystallographic relationship with the GaAs NW body, where $\mathrm{Ga}_{0.82} \mathrm{Mn}_{0.18}$ (100) lies on cubic GaAs (111) or hexagonal GaAs (0001) and $\mathrm{Ga}_{0.82} \mathrm{Mn}_{0.18}$ 


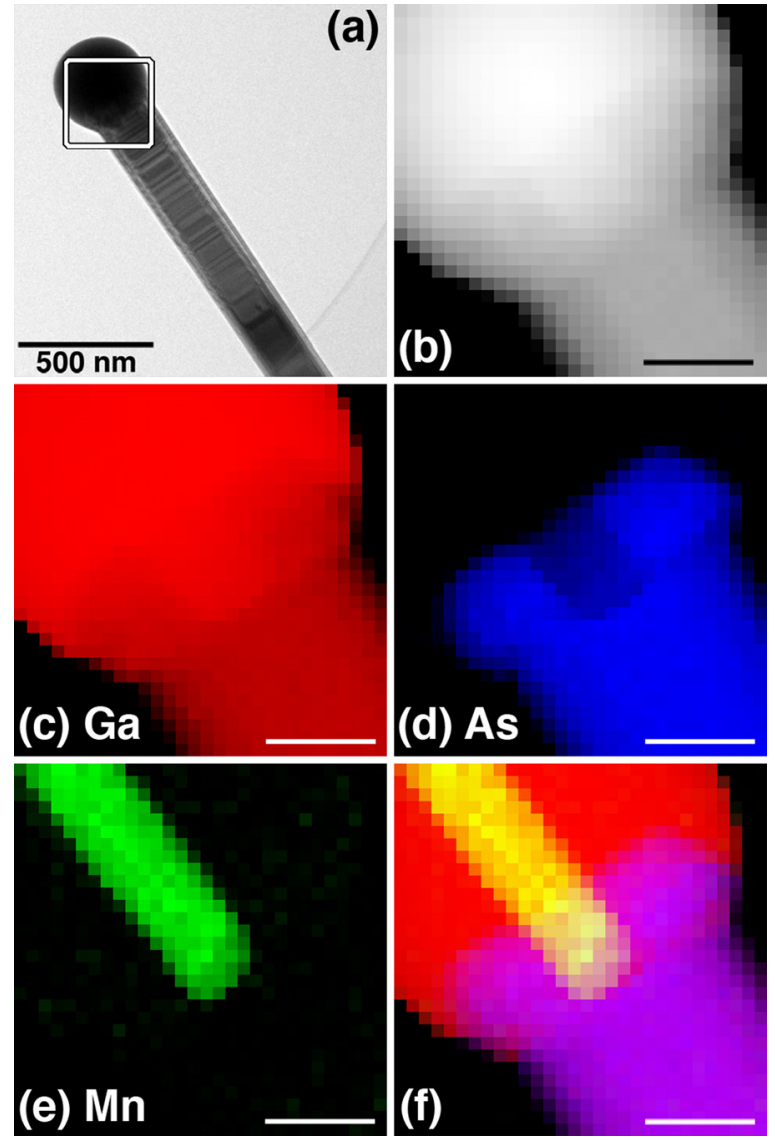

FIG. 2. (a) TEM and (b) STEM-HAADF images acquired from a nanowire taken from sample NW2. (c)-(e) Corresponding quantitative STEM-EDS elemental maps for (c) Ga, (d) As, and (e) Mn, after the application of $\mathrm{PCA}^{24}$ and pixel-by-pixel background subtraction to the spectra. The maximum compositional values for Ga, As, and Mn, respectively, are 100, 50.6, and 3.9 in at. \%. The STEM image and the elemental maps were obtained from the region marked in (a). (f) Composite elemental map formed from images (c)-(e), showing Ga in red, As in blue, and Mn in green. In the composite map, the body of the NW appears purple, while the (Ga,Mn)-containing nanocrystal in the droplet appears yellow. The scale bars in (b)-(f) are $100 \mathrm{~nm}$.

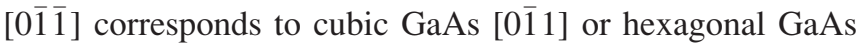
[2110], shown in Fig. 4(f). To the best of our knowledge, this phase has not been reported previously, presumably because such nanocrystals form under specific conditions such as those used here.

An electron tomographic reconstruction of a NW taken from sample NW2 is shown in Fig. 5. The reconstruction reveals the three-dimensional morphology of the $\mathrm{Ga}_{0.82} \mathrm{Mn}_{0.18}$ nanocrystal in the Ga droplet at the end of the NW. It also shows that the nanocrystal is partially embedded in the GaAs NW body and in contact with the bottom surface of a neck region at the end of the NW body. This observation is consistent with the EDS elemental maps shown in Fig. 2 and the STEM images shown in Figs. 4(a) and 4(e).

NW necks are often created after the sources of target elements in the MBE were switched off, allowing postgrowth with residual gases remaining in the growth chamber. $^{35,36}$ The decrease in an As flux during the cooling-down process affects the morphology of the end of NWs and promotes the growth of a zinc-blende GaAs phase. ${ }^{36}$ Our TEM
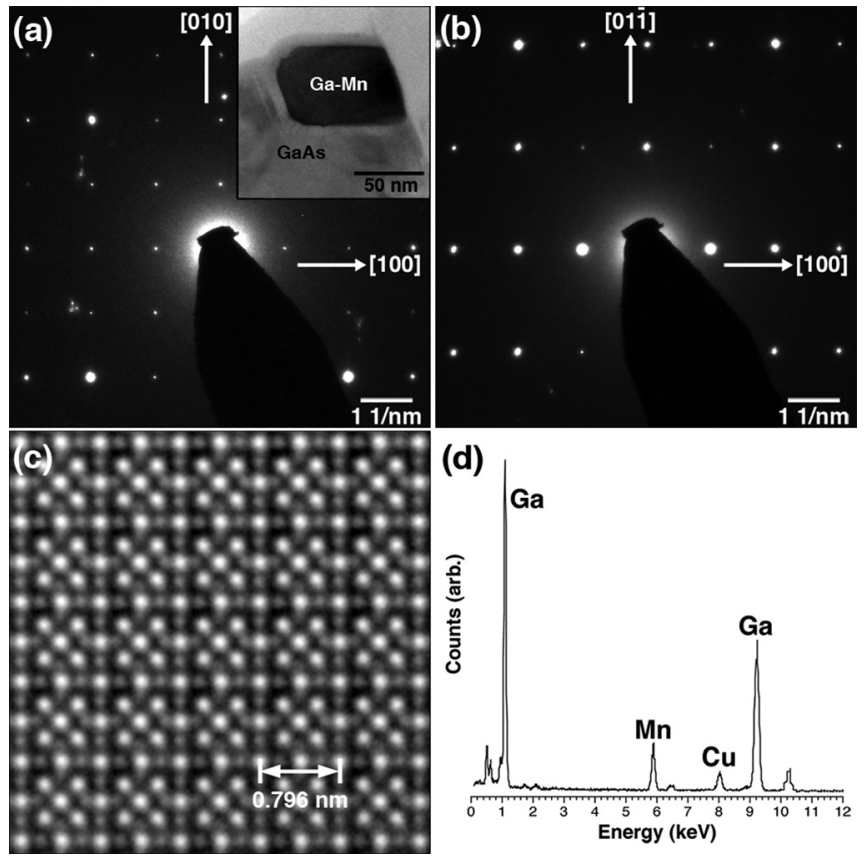

FIG. 3. (a) and (b) Selected-area electron diffraction patterns acquired from an individual (Ga,Mn)-containing nanocrystal in sample NW1, which was prepared for TEM examination using FIB milling and is shown in the inset to (a). The diffraction patterns shown in (a) and (b) were acquired along $\mathrm{Ga}_{0.82} \mathrm{Mn}_{0.18}$ [001] and [011], respectively. (c) High-resolution STEMHAADF image of the $\mathrm{Ga}_{0.82} \mathrm{Mn}_{0.18}$ nanocrystal acquired along its [001]. The details of the atomic arrangement in the crystal are beyond the scope of the present paper. (d) EDS spectrum measured from the same nanocrystal, showing only $\mathrm{Mn}$ and $\mathrm{Ga}$ peaks from the sample and a $\mathrm{Cu}$ signal from the TEM grid used.

observations show that GaAs in the neck region, which has mostly the zinc-blende structure shown in Fig. 4(c), grows continuously from the GaAs NW body (Fig. 4(d)). Compared to the NW body, however, the GaAs in the neck has few twins or stacking faults in the NW growth direction and has a few twin boundaries developing in the direction perpendicular roughly to the NW growth, suggesting that the $\mathrm{GaAs}$ in the neck is likely to form in different conditions from that in the NW body (Figs. 4(b)-4(d)). The GaAs in the neck appears to have formed epitaxially from several nucleation sites at the droplet/NW interface. Consequently, the $\mathrm{Ga}_{0.82} \mathrm{Mn}_{0.18}$ nanocrystals, which are present in the necks, did not nucleate during the major NW growth but formed after turning off the reaction gases. The $\mathrm{Ga}_{0.82} \mathrm{Mn}_{0.18}$ nanocrystals are likely to have nucleated at the same time as the growth of the necks began and to have completed their growth before the growth of the necks ceases, since their shapes are well preserved without any growth steps at the level of the top neck surfaces.

The $\mathrm{Ga}_{0.82} \mathrm{Mn}_{0.18}$ nanocrystals are consistently observed to have elongated shapes with diamond-like cross-sections. The elongated shapes of the nanocrystals can be used to understand why EDS elemental mapping has shown different Mn distributions in different NWs (see Figs. 2(f) and 8(b)). The volumes of the nanocrystal and the droplet measured from the tomographic reconstruction shown in Fig. 5 are $\sim 4$ $\times 10^{5}$ and $\sim 8 \times 10^{6} \mathrm{~nm}^{3}$, respectively, with the nanocrystal occupying $\sim 5 \%$ of the volume of the droplet. 


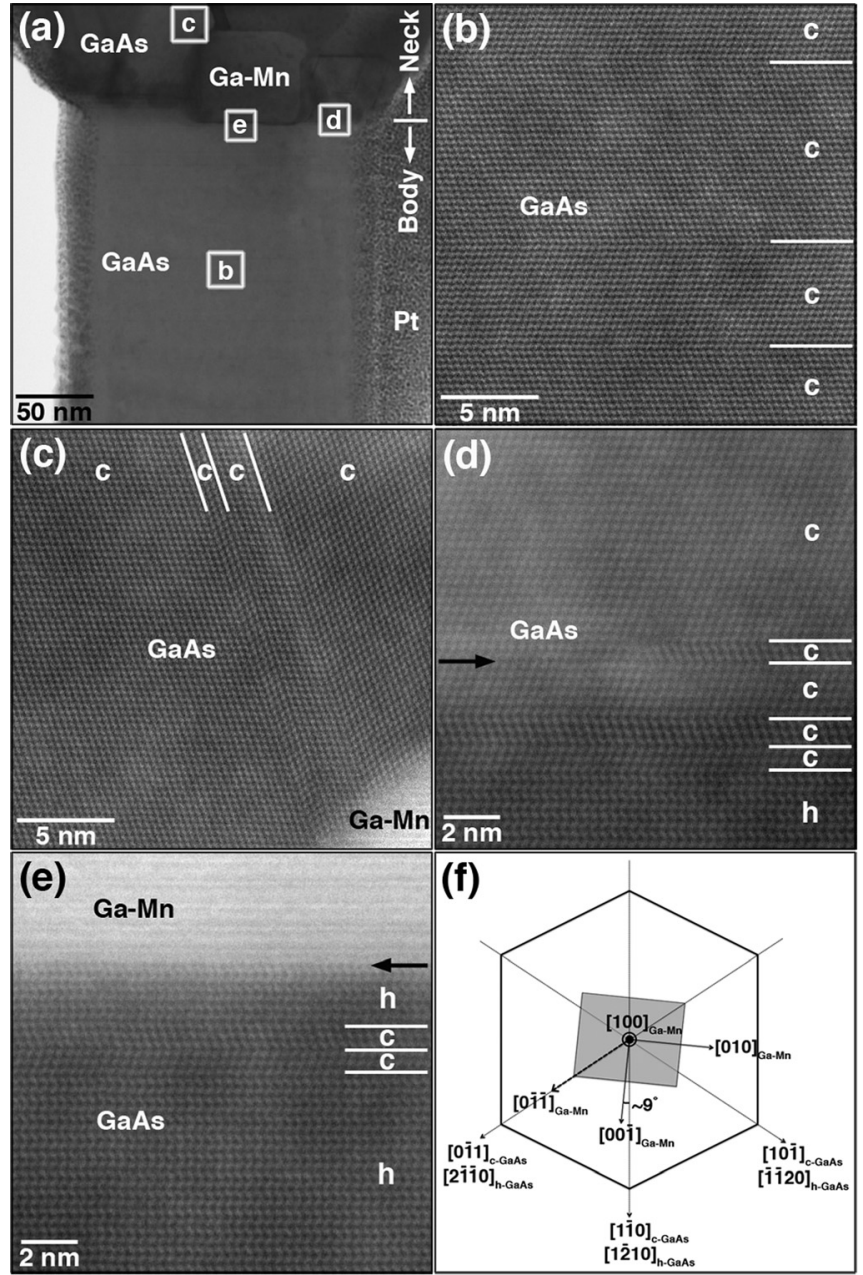

FIG. 4. (a) STEM bright-field and (b)-(e) STEM-HAADF images obtained from a NW in sample NW2. (a) Low-magnification image of the NW, showing a $\mathrm{Ga}_{0.82} \mathrm{Mn}_{0.18}$ nanocrystal (Ga-Mn) lies on the bottom surface of the neck and is surrounded by GaAs. (b)-(e) High-resolution images acquired from the boxes indicated with the letters in (a). The symbols "c" and "h" refer to cubic and hexagonal GaAs, respectively. The viewing direction of GaAs is $[1 \overline{1} 0]_{\mathrm{c}-\mathrm{GaAs}}$ or $[1 \overline{2} 10]_{\mathrm{h}-\mathrm{GaAs}}$. The white lines show twin or phase boundaries, while the black arrows show interfaces between the NW body and the neck (d) or the $\mathrm{Ga}_{0.82} \mathrm{Mn}_{0.18}$ nanocrystal (e). (f) Crystallographic relationship between the $\mathrm{Ga}_{0.82} \mathrm{Mn}_{0.18}$ nanocrystal and GaAs NW body. The angle between $[00 \overline{1}]_{\mathrm{Ga}-\mathrm{Mn}}$ and $[1 \overline{1} 0]_{\mathrm{c}-\mathrm{GaAs}}\left([1 \overline{2} 10]_{\mathrm{h}-\mathrm{GaAs}}\right)$ is about $9^{\circ}$ and the axes of $[0 \overline{1}]_{\mathrm{Ga}-\mathrm{Mn}}$ and $[0 \overline{1} 1]_{\mathrm{c}-\mathrm{GaAs}}\left([2 \overline{1} \overline{1} 0]_{\mathrm{h}-\mathrm{GaAs}}\right)$ correspond to one another.

We propose a formation mechanism for the $\mathrm{Ga}_{0.82} \mathrm{Mn}_{0.18}$ nanocrystals, which is based on VLS growth and illustrated schematically in Fig. 6. The Ga and As that are supplied through the liquid Ga droplet are responsible for the growth of the NW body. Most of the Mn supplied to the $\mathrm{Ga}$ droplet remains within it and is not incorporated into the NW because of its low solubility in GaAs (see LA-APT results below). The $\mathrm{Mn} / \mathrm{Ga}$ atomic ratio in the droplet is estimated to remain below 0.01 , based on the volumes of the droplet and the nanocrystal and their compositions (assuming a density for liquid $\mathrm{Ga}$ of $\left.6.1 \mathrm{~g} / \mathrm{cm}^{3}\right){ }^{37}$ After turning off the $\mathrm{MBE}$ reaction, the temperature decreases gradually from $600{ }^{\circ} \mathrm{C}$ at cooling rates of $30-40^{\circ} \mathrm{C} / \mathrm{min}$ (Fig. 6(b)). There is still a small amount of residual As in the liquid droplet and in the MBE chamber (at the level of $10^{-8} \mathrm{mbar}$ ), which

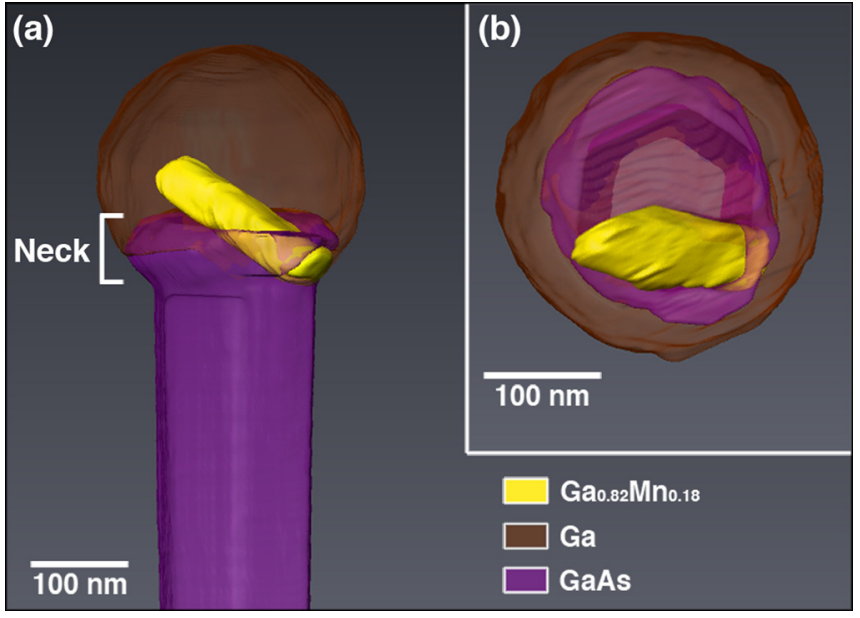

FIG. 5. Isosurface visualization showing (a) side and (b) top views of an electron tomographic reconstruction of a GaMnAs NW taken from sample NW2. The reconstructed volume has been segmented into three separate parts, which are displayed in different colors according to their measured chemical compositions. The elongated $\mathrm{Ga}_{0.82} \mathrm{Mn}_{0.18}$ nanocrystal shown in yellow is in contact with the end of the body of the NW and partially embedded in the GaAs of the neck region.

results in limited further NW growth (i.e., post-growth) and in the formation of a "GaAs neck." A tetragonal $\mathrm{Ga}_{0.82} \mathrm{Mn}_{0.18}$ nanocrystal starts to form at almost the same time, growing with an elongated morphology roughly along its $c$-axis direction until the available $\mathrm{Mn}$ is consumed. The orientation of the nanocrystal depends on the choices of the cubic GaAs \{111\} surfaces to be shared and of the shared axis of $[0 \overline{1} \overline{1}]_{\text {Ga-Mn }}$ with $[0 \overline{1} 1]_{\mathrm{c}-\mathrm{GaAs}}$, allowing 24 possible orientations to the $\mathrm{Ga}_{0.82} \mathrm{Mn}_{0.18}$ nanocrystal. The nanocrystal grows more rapidly than the GaAs in the neck region, which continues to grow after the $\mathrm{Ga}_{0.82} \mathrm{Mn}_{0.18}$ nanocrystal has formed. On the assumption that the GaAs growth rate continues to be $\sim 0.4 \mathrm{~nm} / \mathrm{s}$ (see Table I) and that the length of the neck is $\sim 60 \mathrm{~nm}$ (Fig. 5), the growth of the neck is predicted to take 2-3 min, while the temperature decreases to $480-540{ }^{\circ} \mathrm{C}$. These temperatures are still high enough for the growth of GaAs. ${ }^{2}$ Therefore, the $\mathrm{Ga}_{0.82} \mathrm{Mn}_{0.18}$ nanocrystal is estimated to form at between 480 and $600^{\circ} \mathrm{C}$.

$\mathrm{Ga}_{1-\mathrm{x}} \mathrm{Mn}_{\mathrm{x}}$ As that contains a significant $\mathrm{Mn}$ concentration $(\mathrm{x}>0.01)$ is expected to show a paramagnetic to ferromagnetic phase transition due to the exchange interaction between charge carriers (holes) and localized spins of $\mathrm{Mn}$ ions occupying $\mathrm{Ga}$ sites. ${ }^{38} \mathrm{Mn}$ acts as a shallow acceptor when it substitutes for $\mathrm{Ga}$, providing a high concentration of holes, which are essential for achieving the functionality of DMS. Previous photoluminescence measurements of similar NWs have shown some Mn incorporation into the NWs. ${ }^{20}$ As our TEM-EDS measurements suggest that the Mn concentration in the present NWs is below the detection limit of the technique $(\sim 0.1 \mathrm{wt} . \%)$, the NWs would not be expected to become ferromagnetic. Nevertheless, it is still worth to know the quantitative incorporation amount of $\mathrm{Mn}$ into the individual GaMnAs NWs grown at the present conditions and its distribution.

Low-temperature cathodoluminescence measurements were performed on individual NWs (not shown). As the 
(a)

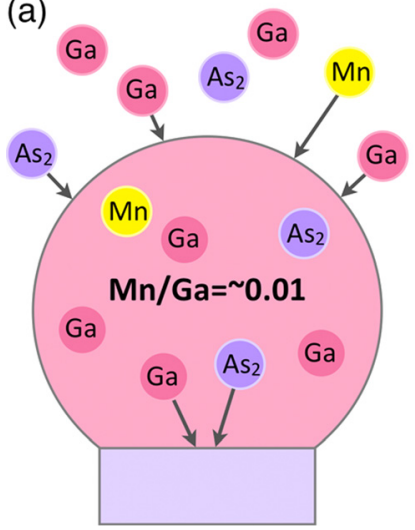

(b)

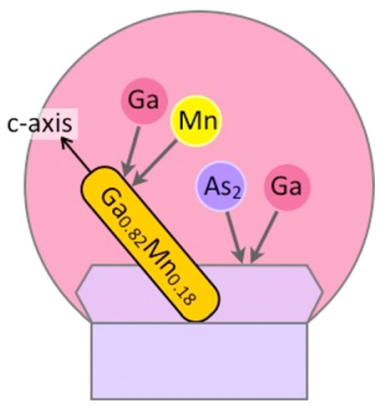

FIG. 6. Schematic diagrams showing the proposed growth mechanism of the $\mathrm{Ga}_{0.82} \mathrm{Mn}_{0.18}$ nanocrystal in the $\mathrm{Ga}$ droplet, shown for an advanced stage of growth of the NW. (a) corresponds to the situation before turning off the deposition process in the MBE chamber (at a temperature of $600^{\circ} \mathrm{C}$ ). (b) corresponds to the situation after turning off the deposition process, when the temperature decreases gradually by approximately $30-40^{\circ} \mathrm{C} / \mathrm{min}$.

NWs are relatively thin and uncapped, the signal intensity is low and the emission is very sensitive to the electron beam. The undoped GaAs NWs (NW3) show two emission peaks, one related to the bandgap of GaAs and one probably related to Si-incorporation from the $\mathrm{Si}$ substrate. The detection of the emission from the single NWs suggests that the NWs have a high degree of crystallinity. In the GaMnAs NWs, Mn-related emission comes mainly from large crystallites at the base of the NWs, reflecting their larger volume, although some weak emission appears from the NWs.

Recently, LA-APT has been used successfully to study the distributions of dopant atoms in semiconductors with close-to-atomic-resolution ${ }^{34,39,40}$ and applied to a thin film of GaMnAs with 3.5 at. \% Mn. ${ }^{41}$ Here, we use LA-APT to elucidate the concentration and distribution of $\mathrm{Mn}$ in the NW bodies. Figure 7(a) shows a mass spectrum measured from the middle of a GaMnAs NW taken from sample NW1, recorded using LA-APT, showing a distinct $\mathrm{Mn}^{+}$peak at 55 $\mathrm{Da}$ alongside the dominant $\mathrm{Ga}^{+}, \mathrm{As}^{+}$, and $\mathrm{As}^{2+}$ peaks. In addition, a very small peak corresponding to $\mathrm{Mn}^{2+}$ (too small to be distinguished in Fig. 7(a)) is present at 27.5 Da. Molecular As peaks are also present outside the range shown, including $\mathrm{As}_{2}{ }^{+}, \mathrm{As}_{3}{ }^{+}$, and $\mathrm{As}_{4}{ }^{+}$. The Mn concentration is measured to be $22 \pm 4$ at. ppm. Assuming that all of the available Mn occupies Ga lattice sites and provides hole carriers, the carrier concentration is $8.8 \times 10^{17} \mathrm{~cm}^{-3}$, which is in good agreement with a value measured from the similar NW samples using photoluminescence spectroscopy. ${ }^{13,22}$ Our assumption that most of the $\mathrm{Mn}$ is located on Ga lattice sites rather than on interstitial sites therefore appears to be reasonable, indicating that the growth temperature used in this study is high enough to suppress the formation of point defects such as As antisites ${ }^{8}$ and Ga vacancies. ${ }^{9}$ Figure 7(b) shows a LA-APT reconstruction of the Mn distribution in the $\mathrm{NW}$, which suggests that the $\mathrm{Mn}$ is distributed randomly within an examined volume of $40 \times 40 \times 270 \mathrm{~nm}^{3}$. The $\mathrm{Mn}$ therefore does not result in the formation of clusters or precipitates, even though the growth conditions used in this
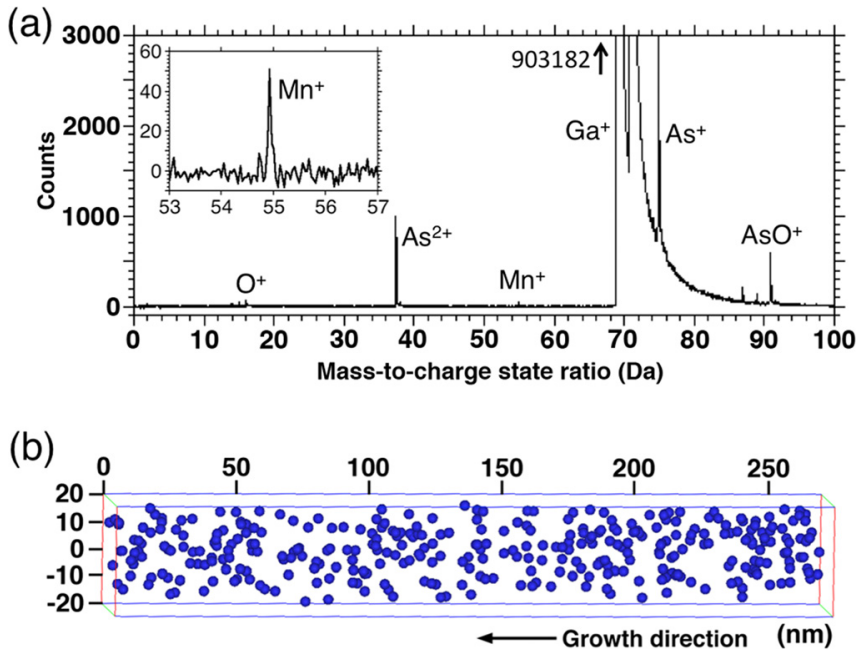

FIG. 7. (a) Part of a mass spectrum recorded from the middle body of an individual GaMnAs NW taken from sample NW1, measured using LAAPT. The total number of atoms counted is $\sim 7 \times 10^{6}$. The number of counts in the $\mathrm{Ga}^{+}$peak is $\sim 9.03 \times 10^{5}$. The experimental parameters used were: laser pulse energy $0.1 \mathrm{~nJ}$; pulse frequency $100 \mathrm{kHz}$; evaporation rate $\sim 0.2 \%$; and temperature $70 \mathrm{~K}$. (b) LA-APT reconstruction of the body of the GaMnAs NW examined in (a), showing a uniform distribution of Mn atoms in a volume of $40 \times 40 \times 270 \mathrm{~nm}^{3}$. The sizes of the individual $\mathrm{Mn}$ atoms are exaggerated for visual purposes.

study can result in segregations of MnAs or As. ${ }^{11,16,17,42}$ Although theoretical calculations have predicted that Mn atoms are localized around the surface of a zinc-blende NW, the tendency was not observed in the volume investigated by LA-APT in this study. ${ }^{43}$ This discrepancy may result from the relatively small cross-section perpendicular to the NW growth direction, $1600 \mathrm{~nm}^{2}$, as compared to a large cross section of $\sim 25000 \mathrm{~nm}^{2}$ (180 nm in thickness) for the entire NW.

Temperature-dependent superconducting quantum interference device (SQUID) measurements obtained from the $\mathrm{Si}$ substrates with the GaMnAs NWs (not shown) indicated the absence of a ferromagnetic phase transition. As the number of NWs contributing to the measurement may not have been sufficient to detect a measurable SQUID signal, we also applied off-axis electron holography to examine magnetic properties of both individual $\mathrm{Ga}_{0.82} \mathrm{Mn}_{0.18}$ nanocrystals and GaMnAs NW bodies (Fig. 8). The technique allows local magnetic fields in materials to be imaged quantitatively at the nanometer scale and has been used extensively for studying the magnetic properties of closely spaced sub-50-nm nanoparticles. ${ }^{26,28,44}$

Figures 8(e) and 8(f) show simulated electron holographic magnetic induction maps of a magnetic nanocrystal in a NW. The geometry used in the simulations is based on the result of the electron tomography shown in Fig. 5. The induction maps were calculated on the assumption of an elongated $\mathrm{Mn}_{1.9} \mathrm{Ga}$ nanocrystal with an induction of $0.38 \mathrm{~T},{ }^{45}$ since magnetic parameters of the new phase $\mathrm{Ga}_{0.82} \mathrm{Mn}_{0.18}$ are not available. The simulated induction maps are predicted to have measurable magnetic dipole fields showing apparent phase contour lines. The difference in the strength of magnetic signals between Figs. 8(e) and 

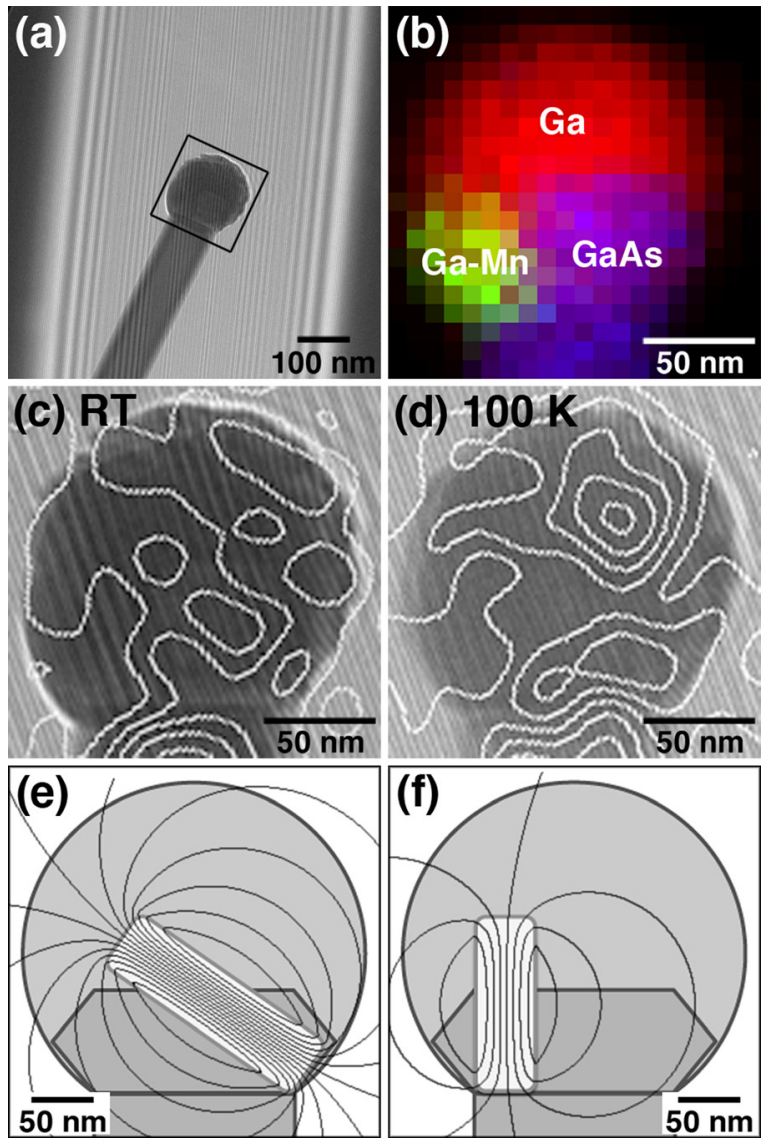

FIG. 8. (a) Off-axis electron hologram recorded from a GaMnAs NW taken from sample NW2. (b) Composite EDS elemental map obtained from the end of the NW. The same colors are used in (b) as in Fig. 2(f). (c) and (d) Remanent magnetic induction maps acquired at (c) room temperature and (d) $100 \mathrm{~K}$ using electron holography from the region marked at the end of the NW in (a). The observed contours are associated primarily with statistical noise and/or artifacts such as changes in contributions to the phase from diffraction contrast between acquiring successive holograms. (e) and (f) Simulated electron holographic magnetic induction maps of a magnetic nanocrystal in a NW. The size, position, and orientation of the nanocrystal are based on the electron tomographic observations shown in Fig. 5. (e) and (f) correspond to viewing directions from the north and east in Fig. 5(b). The magnetic induction of $0.38 \mathrm{~T}$ for a tetragonal $\mathrm{Mn}_{1.9} \mathrm{Ga}$ was used. ${ }^{45}$ The nanocrystal shape was assumed as a cylinder with $180 \mathrm{~nm}$ in length and $50 \mathrm{~nm}$ in diameter. The magnetic phase contour spacing in (c)-(f) is $0.0625 \mathrm{rad}$.

$8(\mathrm{f})$ is due to geometric effect, since electron holography can detect only in-plane magnetic components in specimens.

Figure 8(b) shows an EDS elemental map recorded from the end of a NW taken from sample NW2. Figures 8(c) and 8(d) show corresponding magnetic induction maps acquired using electron holography at room temperature and $100 \mathrm{~K}$, respectively. Although a $\mathrm{Ga}_{0.82} \mathrm{Mn}_{0.18}$ nanocrystal is present in a neck region of the NW, Figs. 8(c) and 8(d) show no convincing magnetic signal, either in the $\mathrm{Ga}_{0.82} \mathrm{Mn}_{0.18}$ nanocrystal or in the NW body, even at the lower specimen temperature. Although some nanoparticles or precipitates can be carriers of magnetization in magnetic semiconductors, ${ }^{16,17}$ our observations suggest that $\mathrm{Ga}_{0.82} \mathrm{Mn}_{0.18}$ does not exhibit a significant magnetic moment at temperatures of $100 \mathrm{~K}$ and above and that it does not contribute measurably to magnetization in the present samples, which is consistent with the SQUID measurements.

\section{CONCLUSIONS}

We have examined GaMnAs NWs grown selfcatalytically at $600^{\circ} \mathrm{C}$ on Si using MBE. Several TEM techniques and LA-APT have been used to determine the distribution of Mn in individual NWs. More than $99 \%$ of the Mn atoms in each NW are found to be accumulated in the form of a tetragonal $\mathrm{Ga}_{0.82} \mathrm{Mn}_{0.18}$ nanocrystal, which forms at the end of the body of the NW after its major growth has stopped. The $\mathrm{Ga}_{0.82} \mathrm{Mn}_{0.18}$ nanocrystals do not have a significant magnetization and are unlikely to contribute to ferromagnetism in other similar systems. A trace amount of $\mathrm{Mn}$ is found to be distributed uniformly in the NW bodies. All of the Mn ions in the NW bodies are likely to occupy Ga sites on the GaAs lattice rather than interstitial sites and to provide hole carriers. The low concentration of $\mathrm{Mn}$ in the NW bodies and the presence of non-magnetic $\mathrm{Ga}_{0.82} \mathrm{Mn}_{0.18}$ nanocrystals provide an explanation for the absence of a ferromagnetic phase transition in the present GaMnAs samples.

\section{ACKNOWLEDGMENTS}

We thank C. B. Boothroyd, T. Dietl, I. Dódony, and K. Stiller for fruitful discussions and A. Kvist for assistance with LA-APT. This work was partly supported by the "FunDMS" Advanced Grant of the European Research Council within the "Ideas" 7th Framework Programme of the European Commission. A.S. acknowledges financial support from the European Commission Network SemiSpinNet (PITN-GA2008-215368) and from the European Regional Development Fund through an Innovative Economy Grant (POIG.01.01.0200-108/09). J.S. acknowledges support by the research Project No. 2014/13/B/ST3/04489 financed through the Polish National Science Centre (NCN). The MBE system in the MAX-IV laboratory was supported through a project granted by the Swedish Research Council (VR). R.D.B. acknowledges financial support from a European Research Council Advanced Grant. The authors acknowledge financial support from the European Union under the Seventh Framework Programme under Contract No. REGPOT-CT-2011-285895Al-NANOFUNC and under a contract for an Integrated Infrastructure Initiative (Reference 312483-ESTEEM2). This study is part of an ongoing collaboration with S. Hata and Mel-Build who provide the HATA holder. The A. P. Møller and Chastine Mc-Kinney Møller Foundation is gratefully acknowledged for their contribution toward the establishment of the Center for Electron Nanoscopy in the Technical University of Denmark.

${ }^{1}$ T. Dietl and H. Ohno, Rev. Mod. Phys. 86, 187 (2014).

${ }^{2}$ H. Ohno, A. Shen, F. Matsukura, A. Oiwa, A. Endo, S. Katsumoto, and Y. Iye, Appl. Phys. Lett. 69, 363 (1996).

${ }^{3}$ M. Wang, R. P. Campion, A. W. Rushforth, K. W. Edmonds, C. T. Foxon, and B. L. Gallagher, Appl. Phys. Lett. 93, 132103 (2008).

${ }^{4}$ H. Ohno, Science 281, 951 (1998).

${ }^{5}$ H.-M. Kim, J.-H. Leem, and T.-W. Kang, Mater. Lett. 52, 39-42 (2002).

${ }^{6}$ M. Lampalzer, S. Nau, C. Pietzonka, W. Treutmann, K. Volz, and W. Stolz, J. Cryst. Growth 272, 772 (2004).

${ }^{7}$ Y. Y. Zhou, X. Liu, J. K. Furdyna, M. A. Scarpulla, and O. D. Dubon, Phys. Rev. B 80, 224403 (2009).

${ }^{8}$ J. Sadowski and J. Z. Domagala, Phys. Rev. B 69, 075206 (2004). 
${ }^{9}$ F. Tuomisto, K. Pennanen, K. Saarinen, and J. Sadowski, Phys. Rev. Lett. 93, 055505 (2004).

${ }^{10}$ K. M. Yu, W. Walukiewicz, T. Wojtowicz, I. Kuryliszyn, X. Liu, Y. Sasaki, and J. K. Furdyna, Phys. Rev. B 65, 201303R (2002).

${ }^{11}$ J. Sadowski, P. Dluzewski, S. Kret, E. Janik, E. Lusakowska, J. Kanski, A. Presz, F. Terki, S. Charar, and D. Tang, Nano Lett. 7, 2724 (2007).

${ }^{12}$ F. Martelli, S. Rubini, M. Piccin, G. Bais, F. Jabeen, S. D. Franceschi, V. Grillo, E. Carlino, F. D’Acapito, F. Boscherini, S. Cabrini, M. Lazzarino, L. Businaro, F. Romanato, and A. Franciosi, Nano Lett. 6, 2130 (2006).

${ }^{13}$ J. Sadowski, A. Siusys, A. Kovács, T. Kasama, R. E. Dunin-Borkowski, T. Wojciechowski, A. Reszka, and B. Kowalski, Phys. Status Solidi B 248, 1576 (2011).

${ }^{14}$ H. S. Kim, Y. J. Cho, K. J. Kong, C. H. Kim, G. B. Chung, and J. Park, Chem. Mater. 21, 1137 (2009).

${ }^{15}$ H. C. Jeon, T. W. Kang, T. W. Kim, Y. J. Yu, W. Jhe, and S. A. Song, J. Appl. Phys. 101, 023508 (2007).

${ }^{16}$ M. Moreno, A. Trampert, L. Däweritz, and K. H. Ploog, Appl. Surf. Sci. 234, 16 (2004).

${ }^{17}$ J. Sadowski, J. Z. Domagala, R. Mathieu, A. Kovács, T. Kasama, R. E. Dunin-Borkowski, and T. Dietl, Phys. Rev. B 84, 245306 (2011).

${ }^{18}$ A. Rudolph, M. Soda, M. Kiessling, T. Wojtowicz, D. Schuh, W. Wegscheider, J. Zweck, C. Back, and E. Reiger, Nano Lett. 9, 3860 (2009).

${ }^{19}$ M. Hilse, Y. Takagaki, J. Herfort, M. Ramsteiner, C. Herrmann, S. Breuer, L. Geelhaar, and H. Riechert, Appl. Phys. Lett. 95, 133126 (2009).

${ }^{20}$ N. S. Dellas, J. Liang, B. J. Cooley, N. Samarth, and S. E. Mohney, Appl. Phys. Lett. 97, 072505 (2010).

${ }^{21}$ A. Šiušys, J. Sadowski, M. Sawicki, S. Kret, T. Wojciechowski, P. Dłużewski, K. Gas, W. Szuszkiewicz, A. Kaminska, and T. Story, Nano Lett. 14, 4263 (2014).

${ }^{22}$ K. Gas, J. Sadowski, T. Kasama, A. Siusys, W. Zaleszczyk, T. Wojciechowski, J.-F. Morhange, A. Altintas, H. Q. Xu, and W. Szuszkiewicz, Nanoscale 5, 7410 (2013).

${ }^{23}$ A. Patsha, S. Amirthapandian, R. Pandian, S. Bera, A. Bhattacharya, and S. Dhara, J. Phys. Chem. C 118, 24165 (2014).

${ }^{24}$ M. Watanabe, E. Okunishi, and K. Ishizuka, Microsc. Anal. 23, 5 (2009).

${ }^{25}$ S. Hata, H. Miyazaki, S. Miyazaki, M. Mitsuhara, M. Tanaka, K. Kaneko, K. Higashida, K. Ikeda, H. Nakashima, S. Matsumura, J. S. Barnard, J. H. Sharp, and P. A. Midgley, Ultramicroscopy 111, 1168 (2011)

${ }^{26}$ R. E. Dunin-Borkowski, T. Kasama, A. Wei, S. L. Tripp, M. J. Hÿtch, E. Snoeck, R. J. Harrison, and A. Putnis, Microsc. Res. Tech. 64, 390 (2004).
${ }^{27}$ M. Beleggia and Y. Zhu, Philos. Mag. 83, 1045 (2003).

${ }^{28}$ T. Kasama, P. Barpanda, R. E. Dunin-Borkowski, S. B. Newcomb, M. R. McCartney, F. J. Castaño, and C. A. Ross, J. Appl. Phys. 98, 013903 (2005).

${ }^{29}$ T. Kasama, R. J. Harrison, N. S. Church, M. Nagao, J. M. Feinberg, and R. E. Dunin-Borkowski, Phase Transitions 86, 67 (2013).

${ }^{30}$ F. Glas, J. C. Harmand, and G. Patriarche, Phys. Rev. Lett. 99, 146101 (2007).

${ }^{31}$ D. Spirkoska, J. Arbiol, A. Gustafsson, S. Conesa-Boj, F. Glas, I. Zardo, M. Heigoldt, M. H. Gass, A. L. Bleloch, S. Estrade, M. Kaniber, J. Rossler, F. Peiro, J. R. Morante, G. Abstreiter, L. Samuelson, and A. Fontcuberta i Morral, Phys. Rev. B 80, 245325 (2009).

${ }^{32}$ G. E. Cirlin, V. G. Dubrovskii, Y. B. Samsonenko, A. D. Bouravleuv, K. Durose, Y. Y. Proskuryakov, B. Mendes, L. Bowen, M. A. Kaliteevski, R. A. Abram, and D. Zeze, Phys. Rev. B 82, 035302 (2010).

${ }^{33}$ B. Mandl, J. Stangl, E. Hilner, A. A. Zakharov, K. Hillerich, A. W. Dey, L. Samuelson, G. Bauer, K. Deppert, and A. Mikkelsen, Nano Lett. 10, 4443 (2010).

${ }^{34}$ D. E. Perea, E. R. Hemesath, E. J. Schwalbach, J. L. Lensch-Falk, P. W. Voorhees, and L. J. Lauhon, Nat. Nanotechnol. 4, 315 (2009).

${ }^{35}$ A. I. Persson, M. W. Larsson, S. Stenström, B. J. Ohlsson, L. Samuelson, and L. R. Wallenberg, Nat. Mater. 3, 677 (2004).

${ }^{36}$ Y. H. Kim, D. W. Park, and S. J. Lee, Appl. Phys. Lett. 100, 033117 (2012).

${ }^{37}$ W. H. Hoather, Proc. Phys. Soc. 48, 699 (1936)

${ }^{38}$ T. Dietl, H. Ohno, F. Matsukura, J. Cibert, and D. Ferrand, Science 287, 1019 (2000)

${ }^{39}$ T. F. Kelly, D. J. Larson, K. Thompson, R. L. Alvis, J. H. Bunton, J. D. Olson, and B. P. Gorman, Annu. Rev. Mater. Res. 37, 681 (2007).

${ }^{40}$ D. E. Perea, J. E. Allen, S. J. May, B. W. Wessels, D. N. Seidman, and L. J. Lauhon, Nano Lett. 6, 181 (2006).

${ }^{41}$ M. Kodzuka, T. Ohkubo, K. Hono, F. Matsukura, and H. Ohno, Ultramicroscopy 109, 644 (2009).

${ }^{42}$ A. Kovács, J. Sadowski, T. Kasama, M. Duchamp, and R. E. DuninBorkowski, J. Phys. D: Appl. Phys. 46, 145309 (2013).

${ }^{43}$ M. Galicka, R. Buczko, and P. Kacman, Nano Lett. 11, 3319 (2011).

${ }^{44}$ T. Kasama, M. Pósfai, R. K. K. Chong, A. P. Finlayson, P. R. Buseck, R. B. Frankel, and R. E. Dunin-Borkowski, Am. Mineral. 91, 1216 (2006).

${ }^{45}$ Y. Huh, P. Kharel, V. R. Shah, X. Z. Li, R. Skomski, and D. J. Sellmyer, J. Appl. Phys. 114, 013906 (2013). 